\title{
Neural Circuits Mediate Electrosensory Behavior in Caenorhabditis elegans
}

\author{
Christopher V. Gabel, ${ }^{1}$ Harrison Gabel,,${ }^{2,3}$ Dmitri Pavlichin, ${ }^{1}$ Albert Kao, ${ }^{1}$ Damon A. Clark, ${ }^{1}$ and \\ Aravinthan D. T. Samuel ${ }^{1}$ \\ ${ }^{1}$ Department of Physics and Center for Brain Science, Harvard University, Cambridge, Massachusetts 02138, ${ }^{2}$ Department of Genetics, Harvard Medical \\ School, and ${ }^{3}$ Department of Molecular Biology, Massachusetts General Hospital, Boston, Massachusetts 02114
}

The nematode Caenorhabditis elegans deliberately crawls toward the negative pole in an electric field. By quantifying the movements of individual worms navigating electric fields, we show that $C$. elegans prefers to crawl at specific angles to the direction of the electric field in persistent periods of forward movement and that the preferred angle is proportional to field strength. C. elegans reorients itself in response to time-varying electric fields by using sudden turns and reversals, standard reorientation maneuvers that $C$. elegans uses during other modes of motile behavior. Mutation or laser ablation that disrupts the structure and function of amphid sensory neurons also disrupts electrosensory behavior. By imaging intracellular calcium dynamics among the amphid sensory neurons of immobilized worms, we show that specific amphid sensory neurons are sensitive to the direction and strength of electric fields. We extend our analysis to the motor level by showing that specific interneurons affect the utilization of sudden turns and reversals during electrosensory steering. Thus, electrosensory behavior may be used as a model system for understanding how sensory inputs are transformed into motor outputs by the $C$. elegans nervous system.

Key words: C. elegans; electrical stimulation; motor control; movement (motion; motor activity); navigation; sensorimotor

\section{Introduction}

The major function of the nervous system is to transform sensory inputs into motor outputs, ranging in sophistication from simple reflexive responses to navigation in variable environments. In a few cases, it has been possible to map feedforward circuits that encode reflexive responses, such as gill withdrawal in Aplysia or touch avoidance in the nematode (Kupfermann and Kandel, 1969; Chalfie et al., 1985). However, the neural circuits that guide navigation are less understood, because complexities may arise at each level from sensory input to motor output. For instance, at the sensory level, an organism may have to integrate time-varying sensory inputs with its own movements to infer its own orientation; an example is Escherichia coli chemotaxis, in which the bacterium processes temporal changes in ambient chemical concentration during periods of its own forward movement to decide whether it is swimming toward or away from nutrients (Berg and Brown, 1972). At the motor level, an organism may have to decide between different types of movements in response to the same sensory inputs; an example is the leech for which triggering movement necessitates the choice between crawling movement or swimming movement (Esch et al., 2002).

Received Feb. 20, 2007; revised June 4, 2007; accepted June 4, 2007.

This work was supported by the Sloan, McKnight, and National Science Foundations. We thank Winfield Hill (Rowland Institute at Harvard University, Cambridge, MA) for electronics, Gary Ruvkun for lending molecular biological resources to this effort, Cori Bargmann for suggesting the connection to parasitism, and Cynthia Chi and Tolulope Adesiyun for help with data collection.

Correspondence should be addressed to Dr. Aravinthan D. T. Samuel, Department of Physics and Center for Brain Science, Harvard University, 17 0xford Street, Cambridge, MA 02138. E-mail: samuel@physics.harvard.edu. DOI:10.1523/JNEUROSCI.0775-07.2007

Copyright $\odot 2007$ Society for Neuroscience $\quad$ 0270-6474/07/277586-11\$15.00/0
The neural basis of navigational behavior may be approachable in the nematode Caenorhabditis elegans, as sensory detection, behavioral choice, and motor output are regulated by a small 302-neuron nervous system. In isotropic environments, $C$. elegans motility may be characterized as periods of forward movement (runs) that are interrupted by reorientation maneuvers (turns and reversals) (Croll, 1975). C. elegans navigates sensory gradients during chemotaxis or thermotaxis based on random trial-and-error (Pierce-Shimomura et al., 1999; Ryu and Samuel, 2002). When the worm happens to be oriented toward improving conditions, it prolongs runs. When it happens to be oriented toward declining conditions, it shortens runs. This strategy is called the biased random walk. In certain environments, $C$. elegans is also capable of direct steering. For example, when the worm navigates thermal gradients at temperatures near its previous cultivation temperature, it tends to crawl along isotherms in single prolonged runs (Hedgecock and Russell, 1975; Luo et al., 2006).

Here, we focus on electrosensory behavior, another case of direct steering. Sukul and Croll (1978) noted the strikingly straight trajectories of wild-type C. elegans as they crawled toward the negative poles of an electric field, but they neither quantified the physical determinants of electrotaxis (also called galvanotaxis) nor determined whether electrotaxis has a neural basis. In this study, we analyzed the movements of individual worms navigating fixed and time-varying electric fields across a wide range of stimulus parameters, quantified the effects of specific genetic and physical perturbations to the $C$. elegans nervous system on electrosensory behavior, and quantified electrosensory detection 
Table 1. Electrotaxis with different types and amounts of ionic salts

\begin{tabular}{lll}
\hline Salt & Concentration $(\mathrm{mm})$ & Slope $\left({ }^{\circ} \mathrm{cm} \mathrm{V}^{-1}\right)$ \\
\hline $\mathrm{NaCl}$ & 0.25 & $4.4 \pm 0.3$ \\
& 1.0 & $4.7 \pm 0.3$ \\
& 4.0 & $4.7 \pm 0.3$ \\
$\mathrm{KCl}$ & 1.0 & $5.2 \pm 0.4$ \\
$\mathrm{CaCl}_{2}$ & 1.0 & $4.1 \pm 0.2$ \\
$\mathrm{NH}_{4} \mathrm{COOH}$ & 1.0 & $4.0 \pm 0.2$ \\
$\mathrm{NaBr}$ & 1.0 & $4.4 \pm 0.2$ \\
\hline
\end{tabular}

The constant of proportionality between approach angle and electric field strength was measured using wild-type worms navigating agar surfaces containing different salt types and concentrations. Absolute approach angles were measured during navigation in voltage gradients between 4 and $14 \mathrm{~V} / \mathrm{cm}$, and the slope \pm SD of linear fits is presented for each case. For $\mathrm{NaCl}$ experiments, each measurement of absolute angle was conducted with 48 worms (these data are also presented in Fig. 10. All other measurements were each conducted with 32 worms. In no case was the proportionality between angle and gradient steepness significantly different from that of $1 \mathrm{~mm} \mathrm{NaCl}$, which we chose for control comparison in these experiments $(p>0.05)$.

among the amphid sensory neurons by imaging intracellular calcium dynamics. Our results establish electrosensory behavior as a new framework for studying how the C. elegans nervous system converts sensory perception into motor behavior.

\section{Materials and Methods}

Strains. C. elegans strains were maintained and grown with standard procedures (Brenner, 1974). We used the wild-type strain N2 and the mutant strains che-1(p674), che-2(e1033), che-13(e1805), eat4(ad819), osm-3 (p802), osm-5 (p813), osm-6(p811), osm-9(ky10), osm-10(n1602), tax-6(p675), tax-4(p678), ttx-1 (p767), ttx-3(ks5), and $o d r-7(k y 4)$, all obtained from the C. elegans Genetics Center (Minneapolis, MN). Transgenic strains $t t x-3:: g f p$ (which labels the AIY neurons), osm6::gfp (which labels the ASJ neurons and others), sra-6::gfp (which labels the ASH and ASI neurons), str-1::gfp (which labels AWB), and che-1(p674);ceh-36::gfp (oy1550) (which labels AWC in a che-1 mutant background) were gifts from P. Sengupta (Brandeis University, Waltham, MA). The transgenic strain $n m r-1:: g f p$, which labels the AVA and RIM neurons, and the mutant strain $g l r-1$ (ky176) dpy-19(n1347ts, mat) III;akIs9 X(Pglr-1::GLR-1(A/T)) were gifts from A. Maricq (University of Utah, Salt Lake City, UT).

Electrotaxis assay. In each experiment, young adult worms were selected from a cultivation plate and rinsed in nematode growth medium buffer. The rinsed worms were then picked to the surface of an agar disc made with deionized water, a salt of defined concentration (see Table 1 for particular salts and concentrations used), glycerol to raise the osmolarity to $50 \mathrm{~mm}$, and $1.7 \% \mathrm{w} / \mathrm{w}$ Bacto-Agar (BD Biosciences, Franklin Lakes, NJ). The agar disc was then placed in the experimental apparatus shown in Figure $1 A$.

The experimental apparatus resembled a two-axis gel electrophoresis chamber. A platform at its center supported the agar disc such that all but its top surface was immersed in a bath solution with the same salt and glycerol concentrations as the disc. The bath was continuously circulated throughout the chamber to reduce resistive heating. During each experiment, we verified that the agar surface temperature did not rise $>1{ }^{\circ} \mathrm{C}$ with a T-type thermocouple.

Voltage across each axis of the chamber was generated with an RIS496.1 computer-controlled vector amplifier provided by W. Hill (Rowland Institute at Harvard University, Cambridge, MA). By separately controlling the voltage across each axis, electric fields of defined direction and amplitude up to $25 \mathrm{~V} / \mathrm{cm}$ could be imposed on the agar surface. Four platinum measurement electrodes were also inserted into the agar surface to continuously verify electric field direction and amplitude during each experiment.

Worms were monitored as described by Ryu and Samuel (2002). In brief, a CCD camera equipped with a zoom lens was used to image the worms on the agar surfaces illuminated obliquely by a ring of superbright light-emitting diodes (LEDs). Images were captured with a PCI framegrabber (National Instruments, Austin, TX). Worm trajectories were analyzed using LabVIEW (National Instruments) and Matlab (Mathworks, Framingham, MA).
Surgery. Laser ablation of individual neurons was performed using standard procedures (Bargmann and Avery, 1995), except that a cavitydumped Ti:sapphire femtosecond laser (KMLabs, Boulder, CO) was used to deliver the laser pulses instead of the more commonly used nanosecond pulsed dye laser. Neurons were killed in L1 larvae anesthetized with sodium azide. The behavior of surgically operated worms was tested at the young adult stage. Destruction of the targeted neuron was double-checked by microscopy after behavioral assays. Laser surgery was performed on young adult worms using the procedures established by Chung et al. (2006). Briefly, the amphid sensory cells of young adult worms were stained with DiI using standard techniques. The worms were anesthetized with sodium azide, and the labeled dendrite was targeted and severed by brief exposure to the Ti:sapphire femtosecond laser beam. Worms were then recovered $(>10 \mathrm{~h})$ and subjected to behavioral assays.

Fluorescence imaging. Intracellular calcium dynamics were measured using the YC3.60 variant of the calcium-sensitive cameleon protein (Nagai et al., 2004). The YC3.60 open reading frame was cloned into the pPD49.26 vector with the unc-54 3' untranslated region. Expression of YC3.60 was driven by the $o d r-4$ promoter, which was amplified from $C$. elegans genomic DNA using primer sequences $5^{\prime}$-AGCTGACATCTAGATGAGATATGTGTTCCGACAGAGCTG-3' and 5'-TGAAGTCCCCGGGTCCTGGAGTTGGACGTCAAACA-3' ${ }^{\prime}$. We quantified calcium dynamics as changes in ratiometric fluorescence emission between the cyan and yellow fluorescent protein components of cameleon, in the same manner that we described previously (Clark et al., 2006). Here, worms were immobilized on a $2 \%$ agar pad containing $0.05 \%$ tetramisole [an acetylcholine agonist that paralyzes the worms (Knobel et al., 1999)] or immobilized with cyanoacrylate glue (Abbott Laboratories, Abbott Park, IL) such that the tip of the nose was exposed. We found no differences in the recordings of intracellular calcium dynamics with worms immobilized with tetramisole or by gluing. A coverslip was placed over the worm, and the preparation was immersed in solution containing 0.25 $\mathrm{mm} \mathrm{NaCl}$ and $50 \mathrm{~mm}$ glycerol in a plastic chamber. The electric field was applied using four platinum electrodes lining the ends of the chamber, under feedback-control using a computer running LabVIEW (National Instruments). Fluorescence resonance energy transfer (FRET) measurements of the cameleon signal were conducted as described by Clark et al. (2006). The response of an individual neuron was measured in the region of interest surrounding the cell body of specific amphid sensory neurons. Cells were identified based on patterns of cameleon expression under the $o d r-4$ promoter. Mosaic expression of the extrachromosomal array did not allow simultaneous measurement from all odr-4-expressing neurons in all worms. Worms were selected for expression of cameleon in subsets of amphid sensory neurons that facilitated unambiguous cell identification.

\section{Results}

C. elegans crawls toward the negative pole of an electric field

First, we quantified the movements of individual young adult worms navigating agar surfaces while subjected to electric fields with fixed amplitude and direction, using an apparatus that allowed us to control the effects of resistive heating as well as define the chemistry and ionic conductivity of the agar surface (Fig. $1 \mathrm{~A}$ ). Representative tracks made by wild-type worms navigating these electric fields are shown in Figure $1 B$. At a threshold of $\sim 3 \mathrm{~V} / \mathrm{cm}$, the worm crawls deliberately toward the negative pole with stable and persistent trajectories along lines at a specific acute angle to the electric field. Electrosensory navigation is robust, and within seconds of turning on the electric field, nearly every worm on an agar surface is entrained in persistent movement toward the negative pole (supplemental movie, available at www.jneurosci.org as supplemental material). Occasionally, an individual worm crawls in the opposite direction toward the positive pole at fields $<3 \mathrm{~V} / \mathrm{cm}$. Because these approaches to the positive pole were rare and difficult to reproduce ( $<1 \%$ of observations), we did not investigate them more closely. In stronger electric fields, the worm crawls toward the negative pole along lines at larger angles to the electric field. We found that the approach angle is propor- 
tional to electric field strength up to 14 $\mathrm{V} / \mathrm{cm}$ at which the worm crawls at $60^{\circ}$ to the electric field (Fig. 1C).

By controlling the salt concentration and thereby the conductivity of the agar surfaces, we were able to distinguish the effects of current and voltage. We did not use salt concentrations higher than $4 \mathrm{~mm}$, which led to resistive heating by $>0.1{ }^{\circ} \mathrm{C}$, or salt concentrations lower than $0.25 \mathrm{~mm}$, because for unknown reasons, $C$. elegans does not crawl on deionized surfaces. Before activating the electric field in each experiment, we verified that worm motility was superficially normal, which we inferred from observations of normal crawling speed as well as spontaneous turns and reversals. We found that neither substituting different ambient ionic salts nor varying salt concentration significantly alters the constant of proportionality between the approach angle and voltage gradient (Table 1). After verifying the negligible effects of different amounts or types of ambient ions on approach angle, we routinely used $0.25 \mathrm{~mm} \mathrm{NaCl}$ in subsequent experiments.

C. elegans crawls on either its left or right side, so that either its dorsal or ventral side faces the negative pole. In strong fields, an individual worm sometimes zigzags toward the negative pole, appearing to alternate between dorsal and ventral tracks but keeping the same absolute angle to the electric field (supplemental movie, available at www.jneurosci.org as supplemental material). Crawling speed is not affected by the electric field in the range that we studied, but $C$. elegans is quickly paralyzed by electric fields stronger than $14 \mathrm{~V} / \mathrm{cm}$. Electrically induced paralysis is reversible; worms immediately resume crawling after switching off the field.

\section{C. elegans responds to time-varying fields using turns and reversals}

We found that $C$. elegans responds to the slow rotation of an electric field by using slight turns to correct its crawling direction. Steady rotation of an electric field causes the worm to crawl in circles, with faster rotation resulting in tighter circles up to $\sim 10^{\circ}$ s (Fig. $2 \mathrm{~A}$ ). Circling in place allowed us to monitor individual worms for extended periods without having to interrupt the field. Individual worms circle for hours without evidence of adaptation or desensitization. After 1-3 h of continuous circling, worms stop moving altogether, perhaps from exhaustion.

We found that C. elegans responds to step rotation of an electric field by immediately reorienting itself to the new direction. $C$. elegans uses two stereotyped maneuvers to reorient itself during motile behavior (Croll, 1975): (1) turns involving a sudden redirection of forward crawling without pause or slowdown (also called omega turns because the worm's body briefly resembles the letter $\Omega$ ); and (2) reversals involving several seconds of backward movement before the worm resumes forward movement in a new direction. A representative turn and reversal in response to $60^{\circ}$ step rotations of the electric field are shown in Figure $2 \mathrm{~B}$. Thus, step rotation of the electric field necessitates a motor decision,
B

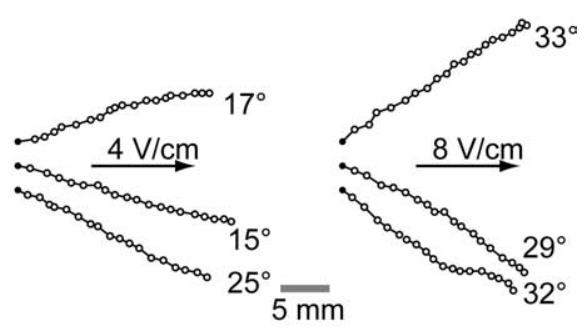

C

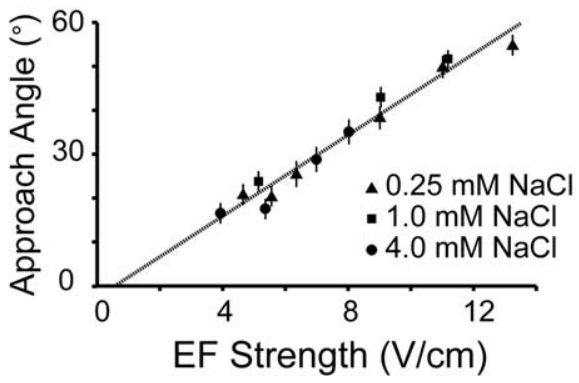

Figure 1. Electrotaxis in fixed electric fields. $A$, Experimental apparatus. Four platinum electrodes (a) lined the sides of a square Absolute angles with respect to the electric field direction are indicated in degrees. $\boldsymbol{C}$, The absolute angle between the

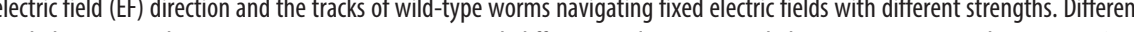
mbols correspond to experiments in environments with different conductivities. Each data point represents the mean $\pm \mathrm{SE}$ of . The slope of the linear fit is $4.6 \pm 0.3^{\circ} \mathrm{cm} \mathrm{V}^{-1}\left(R^{2}=0.963\right)$

whether C. elegans uses a sudden turn or reversal to reorient to the new direction. The choice between turn and reversal appears to be made randomly, but increasing the size of the reorientation angle increases the likelihood of reversal. To reorient by $30^{\circ}$, the worm almost always uses turns. To reorient by $120^{\circ}$, the worm almost always uses reversals (Fig. 2C).

\section{The frequency range of the electrosensory response}

To quantify the frequency range of the electrosensory response, we studied C. elegans subjected to electric fields fixed in direction but modulated in amplitude with sinusoidal waveforms between 0 and $10 \mathrm{~V} / \mathrm{cm}$ at different frequencies. When subjected to modulation frequencies $>16 \mathrm{~Hz}$, C. elegans crawls along straight lines with directions distributed narrowly around a specific absolute angle, just as they would in a fixed field of $5 \mathrm{~V} / \mathrm{cm}$, which corresponds to the DC offset of the modulation. But when subjected to modulation frequencies $<16 \mathrm{~Hz}$, worms fluctuate their crawling direction over a wider range of angles as they appear to sense the modulations in electric field strength and attempt to select new approach angles. Graphing directional fluctuation against the frequency of amplitude modulation, the behavioral response resembles a low-pass filter with a cutoff frequency of $16 \mathrm{~Hz}$ (Fig. $2 D$ ). These data suggest that electrosensation must neither be slower than $16 \mathrm{~Hz}$ nor take longer than $60 \mathrm{~ms}$, a limit within the bounds of C. elegans neurophysiology. Mechanosensory channels of body-wall neurons, for example, conduct current within $5 \mathrm{~ms}$ of light touch (O'Hagan et al., 2005). 

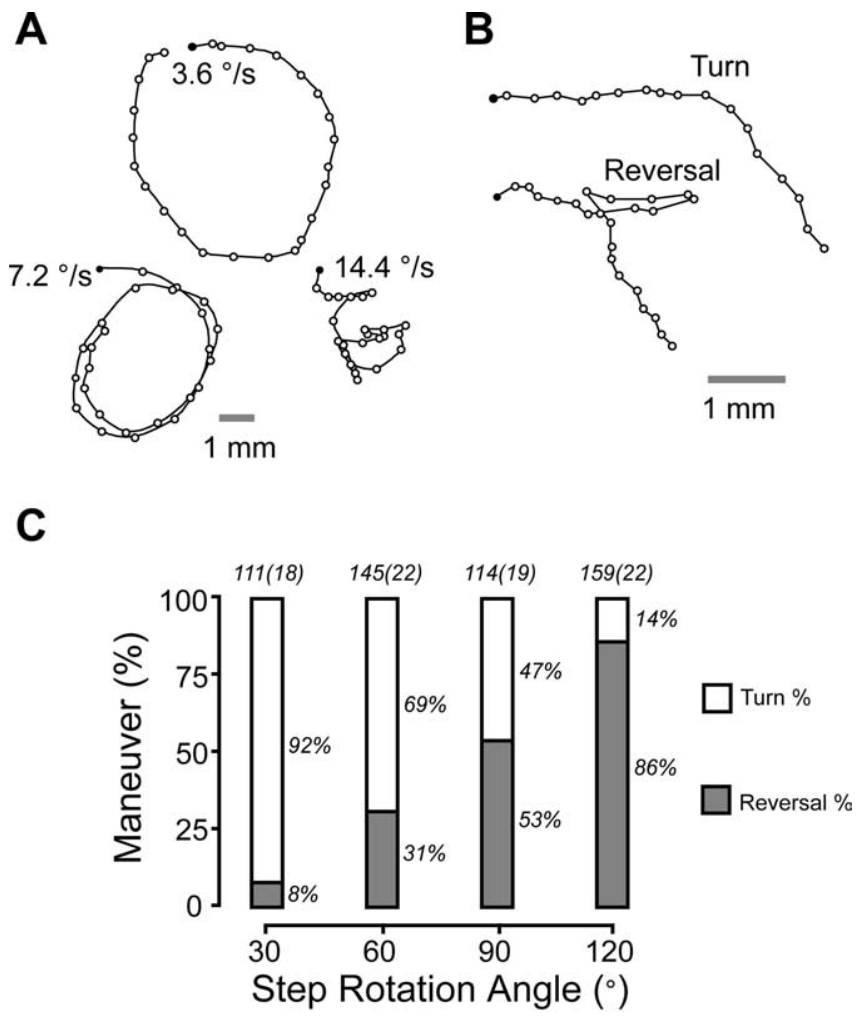

D

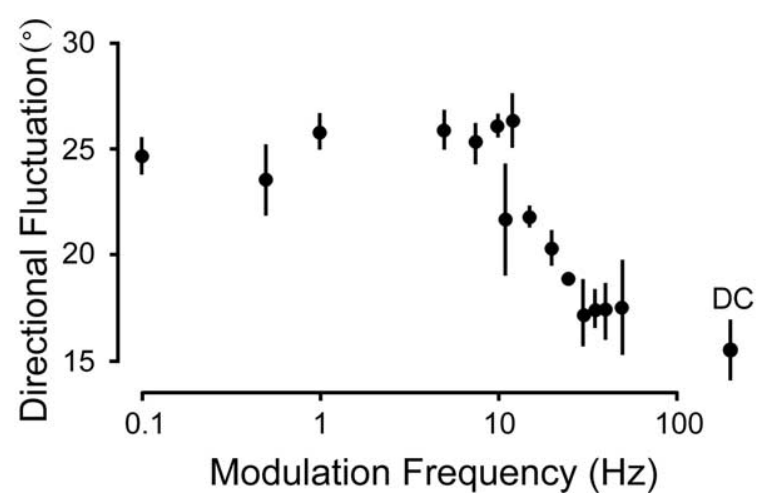

Figure 2. Electrotaxis in time-varying fields. $\boldsymbol{A}$, Representative $100 \mathrm{~s}$ tracks of wild-type worms navigating electric fields with constant strength $(4 \mathrm{~V} / \mathrm{cm})$ but rotating continuously clockwise at $3.6,7.2$, and $14.4 \%$ s. Start positions are indicated with filled circles, and subsequent positions at 4 s intervals are indicated with open circles. In electric fields rotated at 3.6 and $7.2 \%$, worms adjust their crawling direction as quickly as the electric field and thus circle once and twice in $100 \mathrm{~s}$, respectively. Worms subjected to field rotation at $14.4^{\circ} / \mathrm{s}$ cannot keep up with the field and crawl erratically. $\boldsymbol{B}, \mathrm{A}$ representative turn and reversal of wild-type worms subjected to $60^{\circ}$ clockwise step rotation of the electric field. Start positions are indicated with filled circles, and subsequent positions at $1 \mathrm{~s}$ intervals are indicated with open circles. In each case, the step rotation occurred at $t=10 \mathrm{~s}$. The turn was executed without pause or slowdown. The reversal lasted $4 \mathrm{~s}$. C, Percentage use of turn and reversal maneuvers by wild-type worms reorienting themselves in response to step rotations of the electric field. In each trial, the $4 \mathrm{~V} / \mathrm{cm}$ field was fixed for $30 \mathrm{~s}$ to provoke forward crawling before the field was rotated by the set amount. Each worm maneuver in response to each step rotation trial was tallied as a turn or reversal. Each worm would be tested with 5-10 successive trials. The total numbers of trials performed on all worms are indicated above, and the numbers of worms used for each measurement are indicated in parentheses. Because it was difficult to distinguish the ventral and dorsal sides using our microscopy setup (e.g., see supplemental movie, available at www. jneurosci.org as supplemental material), we would alternate clockwise and counterclockwise rotations trial to trial with each worm to average out any systematic dependence on ventral or dorsal orientation. Turns and reversals were distributed over all worms and both directions of rotation (i.e., it was not the case that a percentage of worms always reversed and that a per-

\section{Electrosensory behavior requires the amphid sensory neurons}

The cuticle skin of C. elegans is a dense, protective, collagen-like structure, providing a high-resistance barrier to ionic transport and loss (Johnstone, 1994). Therefore, the sensory neurons that detect electric fields are likely to be those neurons that traverse the cuticle, such as the amphid sensory neurons that detect ambient chemicals. We analyzed electrosensory behavior in various sensory mutants by subjecting individual worms to slowly rotating electric fields $\left(1.5^{\circ} / \mathrm{s}\right)$ and comparing their trajectories to the smoothly circling trajectories of wild-type worms (Fig. $3 A$ ). We characterized the defects of individual worms as either partially defective, meaning that a worm follows the rotating field but with frequent pauses and wrong turns, or severely defective, meaning that a worm is never able to align itself to the rotating electric field within each trial (Fig. 3A). We adopted this strategy for screening electrosensory phenotypes because it is more sensitive to partial defects than, for example, allowing worms to navigate electric fields for an extended period of time and looking for failure to accumulate at the negative pole.

We found that mutations in certain genes that stunt the growth and development of sensory cilia in most or all of the amphid sensory neurons also disrupt the worm's ability to follow rotating electric fields (Fig. 3B). These genes include che-2, che13, osm-3, osm-5, and osm-6, which also affect chemosensory and osmosensory behaviors (Perkins et al., 1986). Each of these genes affect the formation of sensory cilia in the amphid sensory neurons: CHE-2 is a WD40 protein that is required for ciliary extension (Fujiwara et al., 1999); CHE-13 is a transport protein (Haycraft et al., 2003); OSM-3 is an anterograde motor kinesin (Shakir et al., 1993); OSM-5 and OSM-6 are located within the structure of the sensory cilia (Collet et al., 1998; Haycraft et al., 2001). In contrast, genes that affect the development and function of only certain subsets of amphid sensory neurons have lesser or negligible effects on electrosensory behavior. These genes include osm-9 and osm-10, which affect the ASH osmosensory neuron; odr-7, which affects the AWA olfactory neuron; $t t x-1$, which affects the AFD thermosensory neuron; and che-1, which affects the ASE chemosensory neuron (Sengupta et al., 1994; Hart et al., 1999; Satterlee et al., 2001; Uchida et al., 2003).

We also examined several genes that affect signal transduction or synaptic transmission in amphid sensory neuronal pathways. We found that electrosensory behavior is severely disrupted by mutation in the tax-6 gene, which encodes a calcineurin subunit that is widely expressed in neurons and muscle cells, and by mutation in the eat-4 gene, which is required for glutamatergic neurotransmission (Fig. 3B) (Lee et al., 1999; Kuhara et al., 2002). Thus, electrosensory, thermosensory, and chemosensory detection all use amphid sensory neurons as well as common pathways for signal transduction and synaptic transmission.

$\leftarrow$

centage of worms always turned, nor that a worm would always turn toward one direction and reverse toward the other). $\boldsymbol{D}$, The fluctuations in crawling direction of worms navigating electric fields modulated in amplitude between 0 and $10 \mathrm{~V} / \mathrm{cm}$ in sinusoidal waveforms at different frequencies. In each trial lasting $360 \mathrm{~s}$ and with 32 worms, any period of sustained forward crawling $>3 \mathrm{~mm}$ was taken for measurement of its crawling angle with respect to the fixed direction of the negative pole. We quantified the amount of directional fluctuation in each trial as the SD $(\sigma)$ of the distribution of crawling angles. We conducted four trials and report the mean $\pm \mathrm{SE}$ of the four independent measurements of $\sigma$. For comparison, we also present data from experiments in a fixed electric field of $5 \mathrm{~V} / \mathrm{cm}$, which corresponds to the $D C$ offset of these amplitude modulation experiments 
Amphid sensory neurons detect electric field direction and amplitude

To characterize the response properties of sensory neurons that might contribute to electrosensory behavior, we monitored intracellular calcium dynamics in individual amphid sensory neurons of immobilized worms. We prepared transgenic worms that expressed the fluorescent calciumbinding protein cameleon (YC3.60) in all of the amphid sensory neurons under the control of the $o d r-4$ promoter and subjected individual animals to slowly rotating electric fields above the threshold for electrosensory behavior. We found that the ASJ sensory neuron produced the strongest and most reliable stimulusevoked intracellular calcium transients with the rotating electric field (29 stimulus-evoked responses of 34 different worms) (see sample trace in Fig. 4). We also obtained qualitatively similar but smaller stimulus-evoked calcium transients in the ASH, AWB, AWC, and ASK neurons. In contrast, we were unable to detect any stimulus-evoked calcium transients in the ADL and ASI neurons. Interestingly, the strongest intracellular calcium transients were always observed when the immobilized worm was pointing toward the positive pole, not the negative pole. Thus, if electrosensory behavior is regulated by stimulation of amphid sensory neurons, the worm may be directing movement decisions to lower amphid sensory neuronal activity.

To verify that the amphid sensory neurons actually play a functional role in electrosensory behavior, we performed laser ablation experiments. Because more than one amphid sensory neuron might be involved, we first sought to test the involvement of the entire group of amphid neurons. Femtosecond laser ablation now allows us to snip individual nerve fibers in adult C. elegans without disrupting collateral fibers (Chung et al., 2006). We specifically labeled the amphid neurons of individual adult worms using DiI, a red fluorescent membrane dye, and systematically snipped each and every labeled dendrite by exposure to tightly focused femtosecond light pulses emitted from a cavity-dumped Ti:sapphire laser (Fig. 5A). We found that snipping all of the dendrites in both amphid bundles severely disrupted electrosensory behavior (Fig. $5 B$ ).

Next, we assessed the contribution of specific amphid sensory neurons using laser-killing experiments. To facilitate cell identification, we used transgenic lines that expressed green fluorescent protein (GFP) in subsets of amphid sensory neurons, except in the case of ASK laser-killing experiments, in which we used DiI staining to identify the neuron. We ablated targeted neuronal cell bodies in the L1 larval stage and quantified electrosensory behavior in the young adult animals. We found that killing the ASJ or ASH neurons, which had produced the most significant stim-
B
Smooth EF tracking in wild-type worms

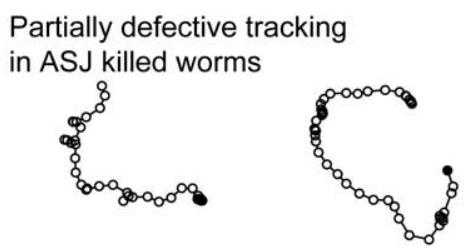

Severely defective EF tracking

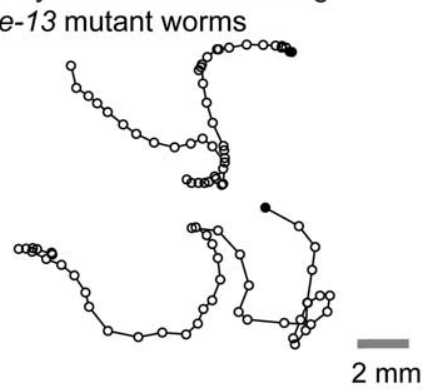

Fraction of trials (\%)

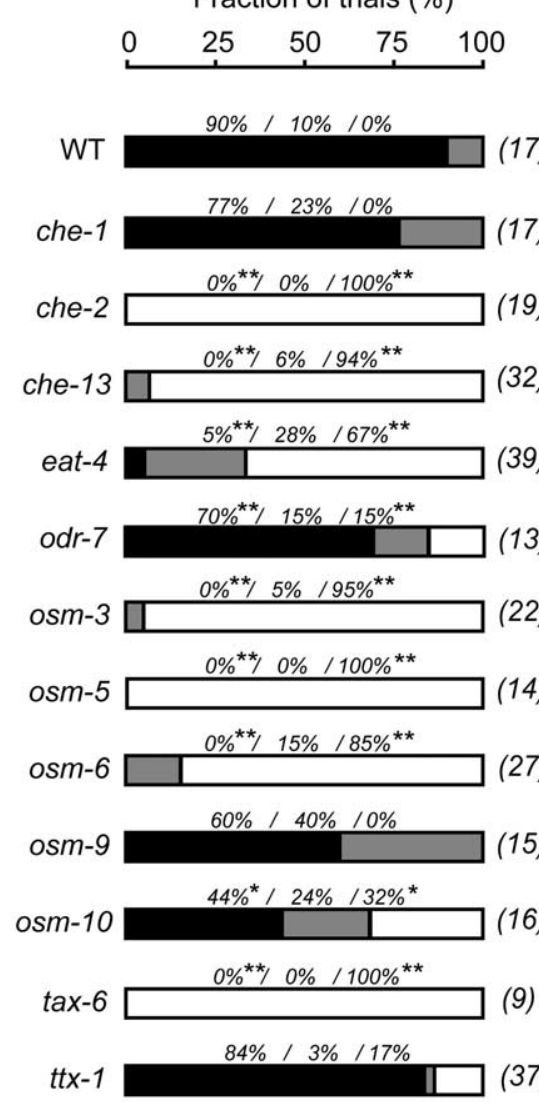

Smooth \% / Partially $\begin{array}{ll}\text { Deverely } \\ \text { Defective } \% ~\end{array}$
Figure 3. Genetic and physical lesions to the amphid sensory neurons affect electrosensory steering. $\boldsymbol{A}$, Representative tracks illustrating the three levels of electrotactic performance of worms subjected to slowly rotating electric fields (EF; $4 \mathrm{~V} / \mathrm{cm}$, rotating Middle, Partially defective tracks generated by worms in which the ASJ neurons have been ablated. These worms effectively follow the electric field, but with limited accuracy, resulting in frequent pauses and wrong turns. Bottom, Severely defective tracks of s the percentage of tracks falling into three categories (smooth, partially defective, and severely defective), as described in $\boldsymbol{A}$. ndividual tracks were scored by eye using the most successful 180 s section of track out of a 300 s trial. Each worm was subjected difference in each of the tallies of smooth, partially defective, and severely defective was calculated compared with the wild-type (WT) value $\left({ }^{*} p<0.05 ;{ }^{* *} p<0.005\right)$.

ulus-evoked calcium dynamics in physiology experiments (Fig. 4), led to significant disruptions in electrotaxis (Fig. 5C). We found that killing neurons that had produced weaker responses in calcium imaging experiments (ASK, AWC, and AWB) had lesser or no effect on electrotactic behavior; it is possible that the smaller contribution of these neurons is masked when ASJ and ASH are intact. When we killed the ASI neuron, from which we were never able to record stimulus-evoked calcium dynamics, we found no behavioral disruption (Fig. 5C). These results suggest that the observed stimulus-evoked calcium dynamics in specific amphid sensory neurons are correlated with behaviorally relevant detection of electric fields.

Physiological response properties of the ASJ sensory neuron We asked whether the physiological response properties of amphid sensory neurons were consistent with quantifiable charac- 


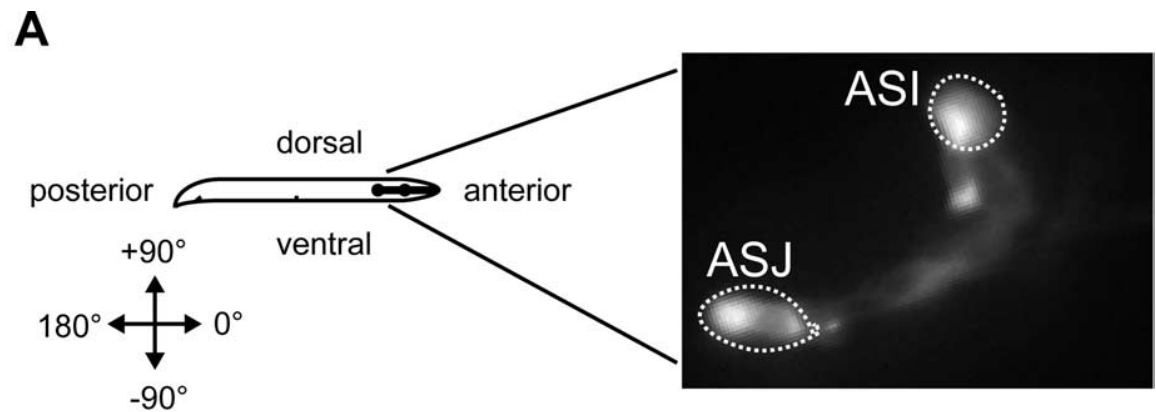

B

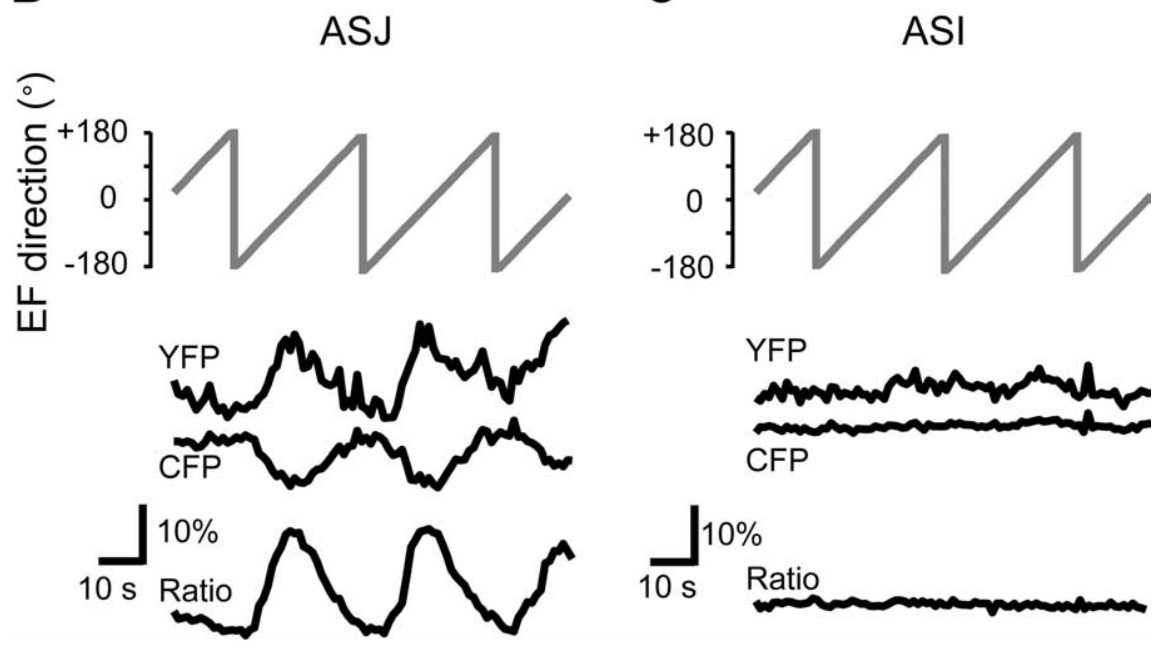

Figure 4. Representative intracellular calcium dynamics in amphid sensory neurons subjected to electric fields. $\boldsymbol{A}$, Representative expression pattern of cameleon under the odr-4 promoter in the worm's head. Specific regions of interest of the fluorescence images were monitored separately to quantify calcium dynamics in separate cells. $\boldsymbol{B}, \boldsymbol{C}$, Worms were immobilized and subjected to a $3 \mathrm{~V} / \mathrm{cm}$ electric field (EF) rotating at $12 \%$. Field direction is defined by the compass and worm schematic shown in $\boldsymbol{A}$. Gray traces plot the direction of the electric field over three complete rotations. The corresponding FRET measurements were calculated as the ratio of yellow (YFP) and cyan (CFP) fluorescence emission from cameleon, based on measurements within the region of interest surrounding individual neurons. A representative FRET response from an ASJ sensory neuron is illustrated in $\boldsymbol{B}$ as well as the lack of a response from an ASI neuron in $\boldsymbol{C}$.

teristics of the navigational behavior. Although all intracellular calcium transients recorded using cameleon from the amphid sensory neurons were qualitatively similar, the consistently largest signals produced by ASJ made it the best choice for more thorough physiological investigation. First, we analyzed directional sensitivity and found that a sinusoidal temporal variation in electric field amplitude (from -3 to $3 \mathrm{~V} / \mathrm{cm}$ ) produced intracellular calcium dynamics that were correlated with electric field direction: the strongest calcium dynamics were obtained when the worm's head was aligned with the electric field and was highest (lowest) when the head was pointing straight toward the positive (negative) pole (Fig. 6A). Another way of analyzing the electric field directional response is to subject the worm to a slowly rotating electric field with fixed amplitude. Using slowly rotating electric fields, we again found that the ASJ neuron is most sensitive to the electric field when the head is pointing directly toward the positive pole (Fig. 6B). Moreover, a 2-3 s delay between the electrical stimulus and the intracellular calcium dynamic response was quantified by using clockwise and counterclockwise rotating electric fields, which produced slight clockwise and counterclockwise shifts, respectively, in the level of intracellular calcium as a function of instantaneous field direction (Fig. 6B). Because behavioral measurements indicate that the electrosensory response may be as fast as $60 \mathrm{~ms}$ (Fig. 2D), it is likely that much of the delay is attributable to slow signal kinetics of the YC3.60 reporter molecule itself (Reiff et al., 2005).

Finally, we asked whether the ASJ neuron exhibited intracellular calcium dynamics in the same amplitude range as the navigational behavior. We fixed the direction of the electric field along the direction of the head of immobilized worms and subjected them to temporal variations in electric field strength using sine waves with different amplitudes (Fig. 6C). In this way, we found that the magnitude of the calcium dynamic response increases in the range of $0-4 \mathrm{~V} / \mathrm{cm}$ and is saturated by stronger electric fields (Fig. 6D).

\section{Neuronal correlates of motor activity}

To obtain evidence that electrosensory behavior is wired into downstream sensorimotor circuits, we examined the contribution of interneurons that are known to affect specific motor outputs. Recently, neural circuits that mediate exploratory navigation in C. elegans have been mapped by identifying specific genetic and physical lesions to the nervous system, affecting the rate of spontaneous turns and reversals exhibited during dispersal in isotropic environments (Tsalik and Hobert, 2003; Wakabayashi et al., 2004; Gray et al., 2005). Although the role of a neuron in the execution of spontaneous maneuvers during dispersal might be different than in that of stimulus-evoked maneuvers during navigation, we took the identification of these neurons as a starting point. In particular, we investigated whether certain neurons might play roles in either the execution of turns and reversals in deliberate reorientations during electrosensory behavior or the suppression of turns and reversals once worms are properly oriented.

Step rotation of the electric field provided a simple assay for the study of turns and reversals in deliberate reorientation. In response to step rotation, wild-type worms use turn and reversal maneuvers randomly, but with quantifiable relative likelihoods (Fig. 2C). We hypothesized that certain neuronal lesions might affect the worm's ability to use turns and reversals when it attempts to reorient itself in response to step rotations of an electric field. The RIM and AVA neurons appeared to be a good starting point for these experiments, because Gray et al. (2005) discovered that killing RIM increases the rate of spontaneous reversals but decreases the rate of spontaneous turns during dispersal in isotropic environments, whereas killing AVA decreases the rate of spontaneous reversals. To facilitate our own RIM and AVA laser killing, we used a transgenic animal that expresses GFP in these neurons. In control experiments with this transgenic animal, we found that $60^{\circ}$ step rotations of the electric field evoked turns and reversals with wild-type proportions but that step rotations would also occasionally evoke stalls. During stalls, worms stop crawling altogether and twitch irregularly in place for $\geq 10 \mathrm{~s}$ before resuming crawling in the new direction. We found that killing RIM or AVA in the transgenic animals led to more stalls. 
Killing RIM reduced the use of turns, killing RIM reduced the use of reversals, and killing both RIM and AVA reduced both the use of turns and reversals. In each case, the increase in stalls was commensurate to the reduction in turns or reversals (Fig. 7A).

To study the suppression of reorientation maneuvers after the worm becomes properly oriented, we tracked worm movement in fixed electric fields. First, we studied the contribution of the AIY neuron, because lesions of AIY have been shown to increase the rates of spontaneous turns and reversals during dispersal in isotropic environments (Tsalik and Hobert, 2003; Wakabayashi et al., 2004; Gray et al., 2005). We used the mutant $t t x-3(k s 5)$ in which AIY does not develop normally (Hobert et al., 1997), and we also laser killed the AIY neurons in a GFPexpressing transgenic strain. We verified previous observations that both the $t+x-3$ mutant and AIY-killed worms exhibit frequent spontaneous reorientation in isotropic environments. In the absence of an electric field, neither worm is able to sustain forward crawls for appreciable distances. But, when exposed to strong electric fields, both worms suppress spontaneous turns and reversals and crawl unmistakably, although more slowly, than wild-type worms, in the direction of the negative pole (Fig. $7 B$ ). A specific mutation of a glutamate receptor, GLR-1(A/T), also increases the rate of spontaneous reorientation in isotropic environments. The GLR-1(A/T) receptor works by depo-

larizing several neurons including AVA and RIM that would express the wild-type receptor (Zheng et al., 1999). Like worms with AIY disruptions, GLR-1(A/T) mutant worms are unable to sustain forward movement in isotropic environments. But placed in an electric field, GLR-1(A/T) mutant worms suppress reorientation maneuvers and crawl toward the negative pole (Fig. 7B).

We also examined whether the AIY interneuron and the GLR$1(\mathrm{~A} / \mathrm{T})$ mutation, because they affect the rate of spontaneous reorientation maneuvers, affect the threshold of electrosensory behavior or the utilization of turns and maneuvers during electrosensory steering. The threshold for electrosensory behavior for wild-type worms is $\sim 3 \mathrm{~V} / \mathrm{cm}$. We found that GLR-1(A/T) worms exhibit nearly the same threshold for electrosensory behavior as wild-type worms. Interestingly, $t t x-3$ mutant worms appear to exhibit a lower threshold, becoming oriented by electric fields as weak as $1.5 \mathrm{~V} / \mathrm{cm}$ (11 of $22 \mathrm{ttx}-3$ mutant worms were oriented by a $1.5 \mathrm{~V} / \mathrm{cm}$ electric field, whereas 0 of 16 wild-type worms were oriented; $p<0.05$ ).

Finally, we studied the use of turns and reversals of AIYdisrupted and GLR-1(A/T) worms during electrosensory steering. In the absence of an electric field, the $t+x$ - 3 mutation not only increases the overall rate of spontaneous reorientation maneuvers but also the fraction that are turns: of spontaneous reorientation maneuvers that are $>45^{\circ}, 30 \%$ are turns for wild type $(n=$
C

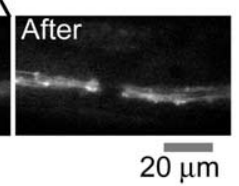

Fraction of trials (\%) $25 \quad 50 \quad 75 \quad 100$ $85 \% / 7 \% / 8 \%$ $0 \% * \pi / 17 \% / 83 \%^{* *}$ (17) \begin{tabular}{c|c} 
Mock surgery \\
AWC killed & $82 \% / 9 \% / 9 \%$ \\
\hline
\end{tabular} (11)
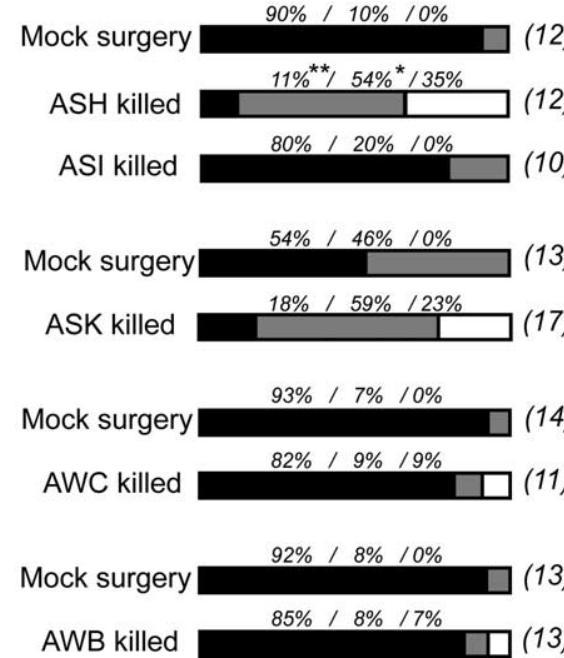

AWB killed emtosec-

ond laser surgery. Amphid sensory neurons of young adult worms were stained using Dil. Individual stained dendrites were

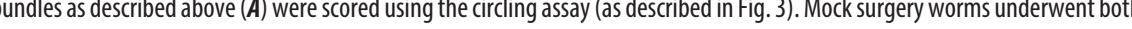
of worms tested is indicated in parenthesis, and $p$ values compare each laser-killing experiment with its corresponding mock surgical control $\left({ }^{*} p<0.05 ;{ }^{* *} p<0.005\right)$

11 worms) and $45 \%$ are turns for $t$ tx-3(ks5) mutants $(n=13$ worms) $(p<0.05)$. In experiments using $60^{\circ}$ step rotations of the electric field, the $t+x-3(k s 5)$ mutation increased the likelihood of stalls, whereas killing AIY reduced the use of reversals, but neither significantly increased the use of turns (Fig. 7A). Thus, lesions to the AIY interneuron appear to have a greater effect on the utilization of sudden turns during spontaneous behavior than during electrosensory steering. The GLR-1(A/T) mutation significantly affects motor choice during electrosensory steering. The GLR$1(\mathrm{~A} / \mathrm{T})$ mutation elevates the rate of spontaneous reversals in isotropic environments. In response to step rotations of the electric field, the GLR-1(A/T) mutant animals are significantly more likely than wild-type worms to use reversals than turns to reorient themselves (Fig. 7A).

\section{A neural circuit for electrosensory behavior}

Our observations show that electrosensory behavior has a neural basis and that its components may be encoded in the hierarchical structure of the nervous system. First, sensory neurons must detect the direction and strength of the electric field. Next, downstream neurons must decide which type of reorientation maneuver to use to correct the worm's alignment. Finally, command motor neurons must execute the motor decisions. Based on the wiring diagram of the C. elegans nervous system (White et al., 1986), we have begun to sketch simple circuits (Fig. 8) that might 
A

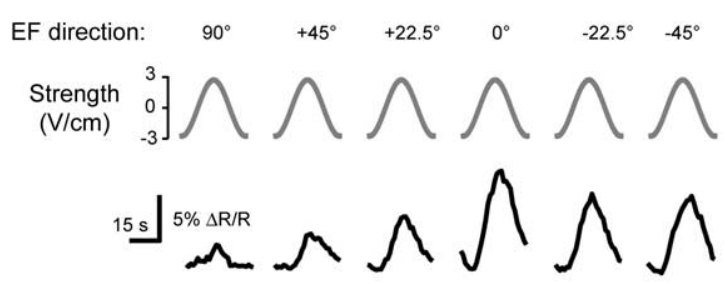

C

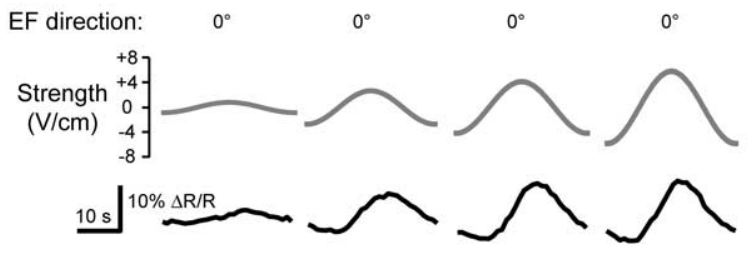

B

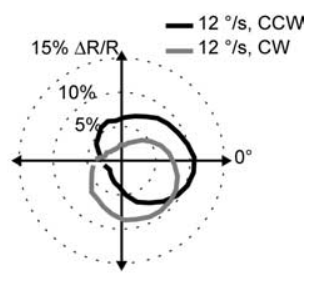

D

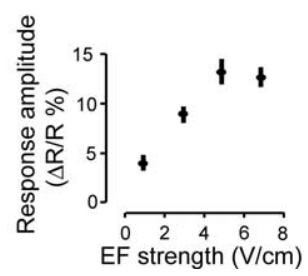

Figure 6. Physiological response of the ASJ sensory neuron. $A$, Directional response of the ASJ sensory neuron. Immobilized worms were subjected to an electric field fixed at specific angles relative to the head $\left(0, \pm 22.5, \pm 45\right.$, and $\left.90^{\circ}\right)$. Field strength was sinusoidally varied between $\pm 3 \mathrm{~V} / \mathrm{cm}$ over a period of $30 \mathrm{~s}$ (gray traces). The FRET measurements from the ASJ neuron at each angle (black traces) is the average of five different ASJ neurons subjected to three oscillations each. The same neurons were used in all six measurements. Electric field directions are defined as in Figure 4. B, Response of the ASJ neuron subjected to a rotating electric field $\left(3 \mathrm{~V} / \mathrm{cm}, 12^{\circ} / \mathrm{s}\right)$. Average FRET measurements are plotted on a polar graph as a function of the electric field direction (angles are measured relative to the worm's head as in Fig. 4). The black trace indicates the response to an electric field that is rotating counterclockwise (CCW), and the gray trace indicates the response to an electric field that is rotating clockwise (CW). The response of the ASJ neuron peaks when the electric field is aligned with the head and decreases monotonically at other angles. A $2-3 s$ delay in the neuronal response is evident from angular shifts in the gray and black traces. Traces are the average response of the same five neurons as in $A$, each subjected to three field rotations. $C$, Response of the ASI neuron to electric fields of different strengths. The direction of the electric field was aligned with the worm's head. Electric field strength was sinusoidally varied with $30 \mathrm{~s}$ period and a series of different amplitudes $( \pm 1, \pm 3, \pm 5$, and $\pm 7 \mathrm{~V} / \mathrm{cm})$. For each measurement, the electric field stimulus is indicated by gray traces, and the black traces represent the average FRET measurements from five ASI neurons subjected to three oscillations. The same five neurons were used for all measurements. $\boldsymbol{D}$, Using the data from $\boldsymbol{C}$, we fit the FRET trace from each neuron for each stimulus strength with a sine function. The response amplitude at each point is the amplitude of these fits averaged across all cells. The response increases monotonically until saturating at $\sim 4 \mathrm{~V} / \mathrm{cm}$. EF, Electric field.

drive electrosensory detection, decision making, and steering. Based on physiological measurements and laser-killing experiments, we suggest that ASJ and ASH play prominent roles in electrosensory detection. We have shown that RIM and AVA contribute to the execution of turns and reversals during electrosensory steering. In Figure 8, we indicate parts of the C. elegans wiring diagram that might connect ASJ and ASH to the RIM and AVA motor neurons during electrosensory behavior. We also include neurons that have been implicated in the execution of turns and reversals during exploratory behavior (Tsalik and Hobert, 2003; Wakabayashi et al., 2004; Gray et al., 2005), alluding to the likely overlap between the neural circuits for electrosensory behavior and other forms of navigational behavior.

Electrosensory behavior is almost certainly distributed over a much larger neural circuit than shown in Figure 8. However, we do suggest that the AIY interneuron, which is excluded from Figure 8, might not contribute significantly to electrosensory behavior. AIY is postsynaptic to chemosensory and thermosensory neurons and has been implicated in the neural circuits that mediate exploratory movement (Tsalik and Hobert, 2003; Wakabayashi et al., 2004; Gray et al., 2005). AIY is not synaptically connected to ASJ or ASH, which had the strongest contributions to electrosensory behavior of the sensory neurons that we studied. The $t t x$-3(ks5) mutation to AIY does not significantly affect electrosensory behavior, except to lower the electric field threshold. One possibility is that in worms with intact AIY interneurons, the response to weak electric fields is actually disrupted by AIY out-

put, because it mediates stochastic movements and exploratory behavior. Thus, removing the AIY interneuron may lower the threshold for electrosensory behavior, as we have observed.

\section{Discussion}

To map navigational behaviors in C. elegans to the structure and function of sensorimotor pathways, it is necessary to develop algorithmic models of behavior, as stepwise transformations of sensory inputs into motor outputs. It is essential to know how specific sensory neurons represent stimulus inputs as patterns in neural activity and how downstream neurons use sensory information to calculate and execute motor decisions. Electrosensory behavior in $C$. elegans provides a powerful system to dissect the sensorimotor circuits that transform a precisely definable sensory input into quantifiable motor output. The extraordinary robustness of electrosensory behavior in C. elegans is striking, given its lack of obvious ecological relevance: C. elegans lives in soil where it is unlikely to encounter strong or stable electric fields. One possibility is that parasitic cousins of $C$. elegans might use electrotaxis when navigating strong electric fields in host tissue. Electric fields within the range of C. elegans electrosensory behavior are commonly found in the organs of larger animals (Nuccitelli, 1992). It has been suggested that Trichinella spiralis, a nematode that preferentially infects skeletal muscles and exhibits electrotaxis in in vitro assays, might detect the difference between different muscle types based on electrosensory cues (Hughes and Harley, 1977). Thus, C. elegans electrosensory behavior may have evolved as a strategy for nematode parasites to exploit directional cues inside their hosts.

For our point of view, electrosensory behavior in C. elegans provides a robust tool for analyzing the function of sensorimotor circuits as they transform a precisely definable sensory input into movement decisions. The quantifiable patterns of electrosensory behavior allow straightforward decomposition of navigation into distinct stages and operations. First, the worm must sense both the direction and magnitude of an electric field. Next, the worm must decide whether to use a turn or reversal to reorient itself to a favorable orientation in the electric field. After actuating the reorientation maneuver, the worm must suppress further reorientation maneuvers to maintain its correct alignment. Using physiological measurements as well as by quantifying the effects of defined genetic and physical lesions to the $C$. elegans nervous system, we should be able to pinpoint how these sensorimotor operations are performed by specific pathways and neural circuits. Here, we discuss these sensorimotor operations and neuronal correlates.

To effect deterministic steering during electrosensory behavior, C. elegans must be able to detect both the direction and amplitude of an electric field. We have shown that a subset of the amphid sensory neurons can detect electric fields in the range of electrosensory behavior and that genetic or laser ablation disrup- 
tion of these neurons reduces the fidelity of electrosensory behavior. Interestingly, these amphid sensory neurons (ASJ and $\mathrm{ASH}$, in particular) are most strongly stimulated when the worm is pointing toward the positive pole. One possibility is that worms orient their movements toward the negative pole in an attempt to lower the activity of these sensory neurons. As far as we know, this is the first demonstration that the ASJ neuron might play a role in navigational behavior. ASJ also responds to environmental cues in the developmental decision to exit the dauerlarval stage (Bargmann and Horvitz, 1991). The ASH neuron has been implicated in several nociceptive reflexes: evoking reversals to avoid nose touch, high osmolarity, and chemorepellants (Hart et al., 1999). Thus, it is interesting to note that ASH-mediated motor decisions, during reflexive avoidance and during electrosensory behavior, may be geared toward minimizing ASH activity.

When the worm detects its own misalignment in an electric field, it executes reorientation maneuvers to correct its alignment. Reorientation necessitates the motor decision to reorient using either a turn or reversal. In the end, either reorientation maneuver achieves the same goal, orienting the worm in the appropriate direction. Choice variability, the production of different, mutually exclusive behaviors when a nervous system is presented with identical stimuli, indicates a simple form of decision making. Another well studied example of choice variability in a sensorimotor system involves the medicinal leech, where stimulation of specific sensory inputs variably evokes swimming or crawling, a behavioral decision that can be biased by the activity of specific neurons (Briggman et al., 2005).

Our results suggest that choice variability in C. elegans electrotaxis, the decision to use either a turn or reversal to reorient in response to an electric field, is wired into specific interneuronal circuits. We suggest that killing either the RIM or AVA interneuron does not bias stimulus-evoked behavioral decision making but does affect the execution of the behavioral decision. Killing RIM, for example, does not affect the fraction of reversals that the worm uses to reorient itself to a step rotation of the electric field. We suggest that the increased numbers of stalls exhibited by RIM-killed worms may represent frustrated attempts to turn by using a missing neuronal pathway. In the same way, killing AVA does not affect the fraction of turns that the worm uses to reorient itself, and the increased numbers of stalls exhibited by AVA-killed worms may represent frustrated attempts to reverse. The simplest interpretation is that removing either the RIM or AVA interneurons does not affect an earlier decision to turn or reverse but does affect the worm's ability to execute either type of maneuver after the decision has been made. Because a portion of turns and reversals are executed normally after RIM or AVA or even both are killed, these neurons are apparently not always required to execute either maneuver. This points to parallel or redundant neural pathways for each maneuver that are capable of functioning correctly without either RIM or AVA. This observation also points to multiple stages in motor decision making. First, the worm has to decide whether to turn or reverse. If it decides to turn, then it has to decide whether to do so by using RIM or an RIM-independent pathway. If it decides to reverse, then it has to decide whether to do so by using an AVA or an AVA-independent pathway. We suggest that successive layers in the hierarchical organization of the worm's nervous system may perform successive stages in motor decision making (Fig. 8).

C. elegans electrosensory behavior has not received the attention that has been given to other navigational behaviors such as chemotaxis or thermotaxis. One reason may be that it was not known whether electrosensory behavior was actually programmed into the C. elegans nervous system. C. elegans is among a wide variety of motile cells and organisms that exhibit electrotaxis. These include bacteria (Adler and Shi, 1988), fungi (Gow, 1994), amoeba (Korohoda et al., 2000), the slime mold Dictyoste- 


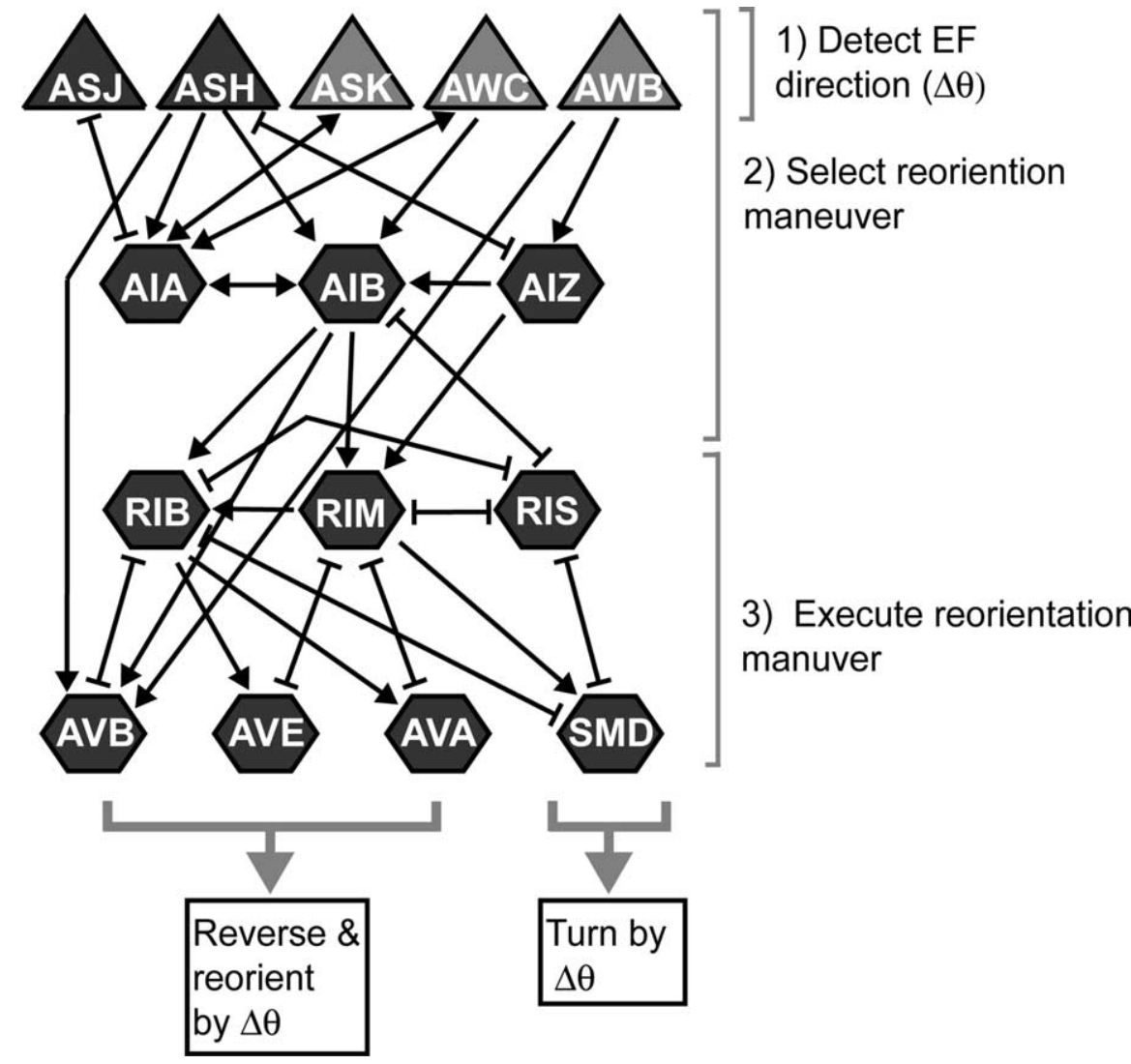

Figure 8. Neural circuits for electrosensory behavior. The wiring diagram of neural pathways that might contribute to electrosensory behavior is shown. Synaptic connections between neurons follow the wiring diagram established by White et al. (1986). Sensory neurons are indicated by red triangles. Interneurons and command motor neurons are indicated by hexagons. Chemical synaptic connections between neurons are indicated by arrows. Gap junctions are indicated by brackets. We suggest that the primary neurons for electrosensory detection are ASJ and ASH. RIM and AVA appear contribute to turns and reversals during electrosensory steering, respectively. Additional neurons show pathways that might connect ASJ, ASH, RIM, and AVA to motor output during electrosensory behavior, as well as several other neurons that have been implicated in the execution of turns and reversals during exploratory behaviors (Tsalik and Hobert, 2003; Wakabayashi et al., 2004; Gray et al., 2005). EF, Electric field.

tem, it should be possible to obtain a complete map of the neural circuits for electrotaxis. Because of recent developments in physiological imaging (Faumont and Lockery, 2006; Clark et al., 2007), it may soon be possible to quantify the operation of these neural circuits within freely moving worms as they navigate electric fields.

\section{References}

Adler J, Shi W (1988) Galvanotaxis in bacteria. Cold Spring Harb Symp Quant Biol 1:23-25.

Bargmann CI, Avery L (1995) Laser killing of cells in Caenorhabditis elegans. Methods Cell Biol 48:225-250.

Bargmann CI, Horvitz HR (1991) Control of larval development by chemosensory neurons in $\mathrm{Cae}$ norhabditis elegans. Science 251:1243-1246.

Berg HC, Brown DA (1972) Chemotaxis in Escherichia coli analysed by three-dimensional tracking. Nature 239:500-504.

Brenner S (1974) The genetics of Caenorhabditis elegans. Genetics 77:71-94.

Briggman KL, Abarbanel HD, Kristan WB (2005) Optical imaging of neuronal populations during decision-making. Science 307:896-901.

Chalfie M, Sulston JE, White JG, Southgate E, Thomson JN, Brenner S (1985) The neural circuit for touch sensitivity in Caenorhabditis elegans. J Neurosci 5:956-964.

Chung SH, Clark DA, Gabel CV, Mazur E, Samuel $\mathrm{AD}$ (2006) The role of the AFD neuron in $C$. elegans thermotaxis analyzed using femtosecond laser ablation. BMC Neurosci 7:30.

Clark DA, Biron D, Sengupta P, Samuel AD (2006) The AFD sensory neurons encode multiple functions underlying thermotactic behavior in Caenorhabditis elegans. J Neurosci 26:7444-7451.

Clark DA, Gabel CV, Gabel H, Samuel AD (2007) Temporal activity patterns in thermosensory neurons of freely moving Caenorhabditis el-

lium discoideum (Zhao et al., 2002), and neuronal growth cones (Rajnicek et al., 1994). In most cases, the biological relevance of electrotaxis is uncertain. However, in many cases, the exquisite control of electrical stimulus inputs has provided a powerful probe of the signaling mechanisms that give rise to directional migration, mechanisms that converge with those of betterstudied behavioral modalities such as chemotaxis. This study establishes electrotaxis in C. elegans as a direct product of its nervous system and as a highly accessible framework for understanding how a simple nervous system transforms patterns of sensory input into motor output during navigational behavior.

Fundamental questions remain. Why does the worm prefer to crawl at a specific angle to the electric field in strong electric fields? The mechanisms of electrosensory detection during navigation may be quite complex. The worm crawls in a snakelike undulating manner, so, in a fixed electric field, as the worm's nose swings from side to side with each undulation, the amphid sensory neurons should be driven into time-varying patterns of activity. The worm may be using the temporal variations in amphid sensory neuronal activity, not just their average level, when calculating movement decisions. To fully understand how the worm calculates movement decisions and executes reorientation maneuvers with precise angular size, we require a more complete map of the relevant sensorimotor circuits. Through systematic genetic and laser ablation analysis of the C. elegans nervous sys- egans encode spatial thermal gradients. J Neurosci 27:6083-6090.

Collet J, Spike CA, Lundquist EA, Shaw JE, Herman RK (1998) Analysis of osm-6, a gene that affects sensory cilium structure and sensory neuron function in Caenorhabditis elegans. Genetics 148:187-200.

Croll NA (1975) Behavioral analysis of nematode movement. Adv Parasitol 13:71-122.

Esch T, Mesce KA, Kristan WB (2002) Evidence for sequential decision making in the medicinal leech. J Neurosci 22:11045-11054.

Faumont S, Lockery SR (2006) The awake behaving worm: simultaneous imaging of neuronal activity and behavior in intact animals at millimeter scale. J Neurophysiol 95:1976-1981.

Fujiwara M, Ishihara T, Katsura I (1999) A novel WD40 protein, CHE-2, acts cell-autonomously in the formation of C. elegans sensory cilia. Development 126:4839-4848.

Gow NA (1994) Growth and guidance of the fungal hypha. Microbiology 140:3193-3205.

Gray JM, Hill JJ, Bargmann CI (2005) A circuit for navigation in C. elegans. Proc Natl Acad Sci USA 430:317-322.

Hart AC, Kass J, Shapiro JE, Kaplan JM (1999) Distinct signaling pathways mediate touch and osmosensory response in a polymodal sensory neuron. J Neurosci 19:1952-1958.

Haycraft CJ, Swoboda P, Taulman PD, Thomas JH, Yoder BK (2001) The C. elegans homolog of the murine cystic kidney disease gene Tg737 functions in a ciliogenic pathway and is disrupted in osm-5 mutant worms. Development 128:1493-1505.

Haycraft CJ, Schafer JC, Zhang Q, Taulman PD, Yoder BK (2003) Identification of CHE-13, a novel intraflagellar transport protein required for cilia formation. Exp Cell Res 284:251-263. 
Hedgecock EM, Russell RL (1975) Normal and mutant thermotaxis in the nematode Caenorhabditis elegans. Proc Natl Acad Sci USA 72:4061-4065.

Hobert O, Mori I, Yamashita Y, Honda H, Ohshima Y, Liu Y, Ruvkun G (1997) Regulation of interneuron function in the C. elegans thermoregulatory pathway by the ttx-3 LIM homeobox gene. Neuron 19:345-357.

Hughes WL, Harley JP (1977) Trichinella spiralis: taxes of first-stage migratory larvae. Exp Parasitol 42:363-373.

Johnstone IL (1994) The cuticle of the nematode Caenorhabditis elegans: a complex collagen structure. BioEssays 16:171-178.

Knobel KM, Jorgensen EM, Bastiani MJ (1999) Growth cones stall and collapse during axon outgrowth in Caenorhabditis elegans. Development 126:4489-4498.

Korohoda W, Mycielska N, Janda E, Madeja Z (2000) Immediate and longterm galvanotactic responses of Amoeba proteus to dc electric fields. Cell Motil Cytoskeleton 45:10-26.

Kuhara A, Inada H, Katsura I, Mori I (2002) Negative regulation and gain control of sensory neurons by the $C$. elegans calcineurin TAX-6. Neuron 33:751-763.

Kupfermann I, Kandel ER (1969) Neuronal controls of a behavioral response mediated by the abdominal ganglion of Aplysia. Science 164:847-850.

Lee RY, Sawin ER, Chalfie M, Horvitz HR, Avery L (1999) EAT-4, a homolog of a mammalian sodium-dependent inorganic phosphate cotransporter, is necessary for glutamatergic neurotransmission in Caenorhabditis elegans. J Neurosci 19:159-167.

Luo L, Clark DA, Biron D, Mahadevan L, Samuel AD (2006) Sensorimotor control during isothermal tracking in Caenorhabditis elegans. J Exp Biol 209:4652-4662.

Nagai T, Yamada S, Tominaga T, Ichikawa M, Miyawaki A (2004) Expanded dynamic range of fluorescent indicators for $\mathrm{Ca}^{2+}$ by circularly permuted yellow fluorescent proteins. Proc Natl Acad Sci USA 101:10554-10559.

Nuccitelli R (1992) Endogenous ionic currents and DC electric fields in multicellular animal tissues. Bioelectromagnetics [Suppl] 1:147-157.

O'Hagan R, Chalfie M, Goodman MB (2005) The MEC-4 DEG/ENaC channel of Caenorhabditis elegans touch receptor neurons transduces mechanical signals. Nat Neurosci 8:43-50.

Perkins LA, Hedgecock EM, Thomson JN, Culotti JG (1986) Mutant sensory cilia in the nematode Caenorhabditis elegans. Dev Biol 117:456-487.

Pierce-Shimomura JT, Morse TM, Lockery SR (1999) The fundamental role of pirouettes in Caenorhabditis elegans chemotaxis. J Neurosci 19:9557-9569.

Rajnicek AM, McCaig CD, Gow NAR (1994) Electric fields induce curved growth of Enterobacter cloacae and Escherichia coli cells: implications for mechanisms of galvanotropism and bacterial growth. J Bacteriol 176:702-713.

Reiff DF, Ihring A, Guerrero G, Isacoff EY, Joesch M, Nakai J, Borst A (2005) In vivo performance of genetically encoded indicators of neural activity in flies. J Neurosci 25:4766-4778.

Ryu WS, Samuel ADT (2002) Thermotaxis in C. elegans analyzed by measuring responses to defined thermal stimuli. J Neurosci 22:5727-5733.

Satterlee JS, Sasakura H, Kuhara A, Berkeley M, Mori I, Sengupta P (2001) Specification of thermosensory neuron fate in C. elegans requires $\mathrm{ttx}-1$, a homolog of otd/Otx. Neuron 31:943-956.

Sengupta P, Colbert HA, Bargmann CI (1994) The C. elegans gene odr-7 encodes an olfactory-specific member of the nuclear receptor superfamily. Cell 79:971-980.

Shakir MA, Fukushige T, Yasuda H, Miwa J, Siddiqui SS (1993) C. elegans osm-3 gene mediating osmotic avoidance behaviour encodes a kinesinlike protein. NeuroReport 4:891-894.

Sukul NC, Croll NA (1978) Influence of potential difference and current on the electrotaxis of C. elegans. J Nematol 10:314-315.

Tsalik EL, Hobert O (2003) Functional mapping of neurons that control locomotory behavior in Caenorhabditis elegans. J Neurobiol 56:178-197.

Uchida O, Nakano H, Koga M, Ohshima Y (2003) The C. elegans che-1 gene encode a zinc finger transcription factor required for specification of the ASE chemosensory neuron. Development 130:1215-1224.

Wakabayashi T, Kitagawa I, Shingai R (2004) Neurons regulating the duration of forward locomotion in Caenorhabditis elegans. Neurosci Res 50:103-111.

White JG, Southgate E, Thomson JN, Brenner S (1986) The structure of the nervous system of the nematode C. elegans. Philos Trans R Soc Lond B Biol Sci 314:1-340.

Zhao M, Pu J, Forrester JV, McCaig CD (2002) Membrane lipids, EGF receptors and intracellular signals co-localize and are polarized in epithelia cells moving directionally in a physiological electric field. FASEB J 16:857-859.

Zheng Y, Brockie PJ, Mellem JE, Madsen DM, Maricq AV (1999) Neuronal control of locomotion in C. elegans is modified by a dominant mutation in the GLR-1 ionotropic glutamate receptor. Neuron 24:347-361. 